Service social

\title{
Mauvais traitements allégués à l'égard d'enfants issus de la diversité ethnoculturelle : nature des signalements à la protection de la jeunesse, de leurs évaluations et typologie des familles signalées
}

\section{Tanya Bousquet Saint-Laurent et Sarah Dufour}

Volume 63, numéro 2, 2017

URI : https://id.erudit.org/iderudit/1046506ar

DOI : https://doi.org/10.7202/1046506ar

Aller au sommaire du numéro

Éditeur(s)

École de travail social et de criminologie de l’Université Laval

ISSN

1708-1734 (numérique)

Découvrir la revue

Citer cet article

Bousquet Saint-Laurent, T. \& Dufour, S. (2017). Mauvais traitements allégués à l'égard d'enfants issus de la diversité ethnoculturelle : nature des signalements à la protection de la jeunesse, de leurs évaluations et typologie des familles signalées. Service social, 63(2), 160-177. https://doi.org/10.7202/1046506ar
Résumé de l'article

Cette recherche qualitative vise une description du contenu des signalements retenus à la protection de la jeunesse ainsi que de leur évaluation à partir des dossiers de 48 situations d'enfants issus de la diversité ethnoculturelle. Ces dossiers ont été sélectionnés par 24 intervenants de la protection de la jeunesse. Les résultats sont organisés selon des thèmes qui suivent de près les critères légaux considérés dans la prise de décision. Un deuxième niveau d'analyse permet de faire ressortir les contenus ethnoculturels ou migratoires dispersés dans les sections narratives et descriptives des dossiers. Par la suite, chaque situation est observée et décrite afin de regrouper des profils familiaux similaires. De cet exercice ressortent quatre portraits familiaux distincts : les familles traversant une crise, les familles qui abdiquent leur rôle parental, les familles isolées et les familles éclatées. 


\title{
Mauvais traitements allégués à l'égard d'enfants issus de la diversité ethnoculturelle : nature des signalements à la protection de la jeunesse, de leurs évaluations et typologie des familles signalées
}

BOUSQUET SAINT-LAURENT, Tanya, M. Sc. psychoéducation

DUFOUR, Sarah, Ph. D. psychologie, professeure agrégée

RÉSUMÉ

Cette recherche qualitative vise une description du contenu des signalements retenus à la protection de la jeunesse ainsi que de leur évaluation à partir des dossiers de 48 situations d'enfants issus de la diversité ethnoculturelle. Ces dossiers ont été sélectionnés par 24 intervenants de la protection de la jeunesse. Les résultats sont organisés selon des thèmes qui suivent de près les critères légaux considérés dans la prise de décision. Un deuxième niveau d'analyse permet de faire ressortir les contenus ethnoculturels ou migratoires dispersés dans les sections narratives et descriptives des dossiers. Par la suite, chaque situation est observée et décrite afin de regrouper des profils familiaux similaires. De cet exercice ressortent quatre portraits familiaux distincts : les familles traversant une crise, les familles qui abdiquent leur rôle parental, les familles isolées et les familles éclatées.

Mots-clés : protection de la jeunesse, signalement, diversité ethnoculturelle, typologie, recherche qualitative.

\begin{abstract}
This qualitative research describes youth protection reports and their assessment based on 48 records of children from diverse ethnocultural backgrounds. These records have been selected by 24 youth protection workers. The results are organized in accordance with themes that are very near the legal criteria taken into consideration towards the decision-making process. A second level of analysis emphasizes ethnocultural or migration related contents appearing in the cases' narrative and descriptive sections. Afterwards, each situation is observed and described so that similar family profiles can be placed together. This exercise yields four distinct family dynamics: families going through a crisis, families abdicating their parenting responsibilities, isolated families, and broken families.
\end{abstract}

Keywords: youth protection intervention, report, ethnocultural diversity, typology, qualitative research. 


\section{INTRODUCTION}

Les recherches au sujet des enfants issus de la diversité ethnoculturelle sont en croissance au Québec (Tardif-Grenier, Lavergne et Sarmiento, 2016) comme ailleurs (Pottie et al., 2015 ; Hirani et al., 2016 ; Millet, 2016), tant en raison de leur poids démographique en constante augmentation et des facteurs de risque qui leur sont propres que d'une volonté affirmée, même légalement comme dans la Loi de la protection de la jeunesse, d'améliorer l'accessibilité et l'adéquation des services. La présente recherche, qualitative, s'intéresse à la nature des allégations de mauvais traitements à l'égard de ces enfants et à leur évaluation par la Direction de la protection de la jeunesse.

\section{Repères conceptuels}

Les connaissances au sujet des enfants issus de la diversité ethnoculturelle, encore disparates, se sont développées en silos, entraînant un foisonnement de concepts et de catégorisations présentant chacun des avantages et des limites. La notion de diversité ethnoculturelle, telle qu'utilisée au Québec et dans le présent texte, se définit comme " une construction sociale qui résulte de rapports sociaux qui se développent en fonction d'un sentiment d'appartenance (réel ou projeté) à un groupe auquel peuvent s'identifier ou non des catégories ethnoculturelles »(Conseil des relations interculturelles, 2007, p. 26). Cette notion vient élargir l'approche centrée sur les migrations, en faisant référence à la cohabitation d'individus et de groupes aux références culturelles diversifiées. Elle est parfois critiquée, car elle peut laisser croire que toutes les cultures de ces groupes sont similaires et qu'elles forment un tout homogène. Elle permet cependant de considérer l'influence de la culture (y compris la langue d'usage, l'appartenance ethnoculturelle et l'appartenance religieuse) dans l'identification et l'explication d'éventuelles disparités, voire discriminations, qui concernent tous ces groupes. Son caractère générique rend possible des déclinaisons à divers contextes. Ainsi, sans faire l'unanimité, la notion de diversité ethnoculturelle présente l'avantage de ne pas enfermer les individus dans des catégories dans lesquelles ils ne peuvent pas se reconnaître ou auxquelles ils n'adhèrent pas. Sont désignées par ce terme générique les personnes immigrantes ou réfugiées, les personnes issues de l'immigration nées au Canada, ainsi que les personnes appartenant aux groupes racisés ou membres de minorités visibles. Selon les sources, diverses manières de désigner les groupes cohabitent dans le présent texte ; il faut y voir un souci de fidélité aux auteurs et non un manque de cohérence.

\section{Diversité ethnoculturelle et maltraitance}

Au Québec, la Loi sur la protection de la jeunesse (LPJ) touche annuellement un nombre considérable d'enfants et de familles. En 2015-2016, pour une population de 1538169 enfants âgés de 0 à 17 ans, 87800 signalements ont été traités, dont 34911 (40\%) ont été retenus pour évaluation (INESSS, 2016). La sécurité ou le développement de l'enfant a été jugé compromis pour 12723 (40\%) d'entre eux. En 2016-2017, pour une population de 358876 enfants montréalais, 9848 signalements ont été traités par le Centre jeunesse de Montréal-Institut universitaire (dont $43 \%$ retenus, soit 4258 signalements). Dans $44 \%$ des évaluations (1 707 cas), le Directeur de la protection de la jeunesse a décidé qu'il y avait compromission (MSSS, 2017).

Les interventions destinées aux enfants en difficulté s'inscrivent dans un contexte de plus en plus diversifié sur le plan culturel, social et religieux. En effet, près du quart de la population montréalaise est immigrante (Statistique Canada, 2013) et $32 \%$ de la population montréalaise est membre d'une 
minorité dite visible, surtout des minorités noires (Ville de Montréal, 2013). Parmi les diverses religions, la religion musulmane est celle qui a connu la croissance relative la plus forte, tendance directement liée à l'immigration (Bibby et Reid, 2015 ; Bibby et Grenville, 2016 ; Ministère de l'Immigration, de la Diversité et de l'Inclusion, 2016). La Commission sur les droits de la personne et de la jeunesse fait état d'une discrimination systémique ayant des répercussions dans l'attitude des services judiciaires, scolaires et de protection de la jeunesse à l'égard des individus différents du groupe majoritaire par "leur couleur de peau, leur apparence ou leur appartenance, réelle ou présumée, à un groupe ethnique " (Eid, Magloire et Turenne, 2011, p. 15). La disproportion des enfants issus de la diversité ethnoculturelle dans les services de protection de la jeunesse est un phénomène étudié tant aux ÉtatsUnis (Hill, 2006 ; Fluke et al., 2011), au Canada (Lavergne et al., 2008) qu'à Montréal (Lavergne et al., 2009 ; Sarmiento et Lavergne, 2017). La représentation de ces enfants aux différentes étapes du processus de protection ne correspond pas à leur représentation dans la population. Des synthèses scientifiques rapportent que les enfants noirs sont souvent surreprésentés à toutes les étapes du processus d'intervention de la protection de la jeunesse et qu'ils demeurent plus longtemps dans les services (Lebrun et al., 2015), alors que les enfants d'autres groupes minoritaires y sont sousreprésentés (Lavergne et al., 2009). Une étude portant sur la distribution géospatiale des taux de signalement à la protection de la jeunesse sur le territoire montréalais montre que le fait d'appartenir à un groupe ethnoculturel fortement minoritaire sur le territoire joue un rôle plus important dans l'explication des signalements des enfants noirs que les facteurs de défavorisation socio-économique traditionnellement associés au risque de maltraitance (Dufour, Lavergne et Ramos, 2015).

Plusieurs pistes explicatives de cette disproportion ainsi que leurs interrelations sont évoquées dans la littérature. Elles se regroupent en trois grands thèmes: 1) des conditions d'adversité plus grandes vécues par les familles ( $p$. ex. : pauvreté, isolement social) ; 2 ) des préjugés culturels ou des pratiques discriminatoires de la part des professionnels de la santé et des services sociaux et 3 ) des facteurs systémiques au sein des organismes communautaires et des services sociaux préventifs, non spécialisés et de protection, entraînant le manque ou l'absence de ressources adéquates (Dezrotes et Portner, 2005 ; Hill, 2006 ; Fluke et al., 2011 ; Dufour, Hassan et Lavergne, 2012 ; Lavergne, Dufour et Couture, 2014).

\section{Processus d'intervention en protection de la jeunesse}

Dans le système québécois, la protection des enfants s'amorce par les inquiétudes d'une personne se trouvant près de la famille et de l'enfant (un professionnel dans l'exercice de ses fonctions, un membre de la famille, l'enfant lui-même ou un citoyen), qui prend la décision de rapporter une situation à la Direction de la protection de la jeunesse (DPJ). Cette première décision d'un signalant est suivie de la première décision de la DPJ, qui consiste à retenir ou non le signalement reçu. Lorsqu'il est retenu, l'intervention de la DPJ se poursuit, soit par l'application de mesures d'urgence (évaluation ultérieure), soit par le processus ordinaire d'évaluation des faits rapportés. À la suite de l'évaluation, l'intervenant détermine si les faits sont avérés et, le cas échéant, s'il y a une situation de compromission ou non. Lorsque la situation est fondée et qu'il y a compromission, l'orientation se fait vers des mesures volontaires ou judiciarisées. La LPJ identifie quatre facteurs d'analyse qui soutiennent les prises de décision: 1) la nature, la gravité, la fréquence et la chronicité des faits signalés, 2) l'âge et les caractéristiques personnelles de l'enfant, 3) la capacité et la volonté des parents de mettre fin à la situation qui compromet la sécurité ou le développement de l'enfant et 4) les ressources du milieu pour 
venir en aide à l'enfant et ses parents (article 38.2 LPJ : Gouvernement du Québec, 2009).

Ces premières étapes et décisions sont déterminantes pour l'enfant et sa famille (primo responsable de l'enfant). Elles conduisent soit à l'amorce d'un parcours dans les services de protection, soit vers une réponse adaptée aux besoins identifiés dans d'autres services que ceux de protection, ou encore à l'absence de réponse (signalement non retenu). Ces décisions reposent sur la reconnaissance des conséquences des mauvais traitements pour les enfants, qui peuvent être très grandes, et même létales (Finkelhor, 2005), ainsi que des conséquences d'une mauvaise réponse aux besoins. En effet, celle-ci peut aussi nuire grandement au fonctionnement familial, entraînant notamment une méfiance accrue envers les autorités professionnelles, une stigmatisation de la famille, des pertes au sein du réseau d'aide, de la détresse psychologique et une perte d'autonomie (Besharov, 2005 ; Hassan et al., 2011).

Cette recherche explore donc en profondeur la nature des signalements à la protection de la jeunesse et leur évaluation pour les enfants issus de la diversité ethnoculturelle. Elle vise d'abord à décrire les signalements ainsi que leurs évaluations selon ce qui est consigné dans les dossiers, afin d'accroître les connaissances sur ces premières étapes décisionnelles. Le deuxième objectif consiste à élaborer, à partir de l'analyse des signalements et des évaluations, une typologie des familles signalées en protection.

\section{MÉTHODE}

Cette étude est menée selon un devis descriptif basé sur une analyse qualitative thématique. II s'agit d'une analyse secondaire (Bousquet Saint-Laurent, 2013) de données recueillies dans le cadre d'une étude antérieure portant sur l'intervention en protection de la jeunesse auprès du groupe des minorités visibles ${ }^{1}$ (voir Dufour et al., 2016, pour la présentation de l'étude originale et ses résultats).

\section{Situations à l'étude}

Vingt-quatre intervenants travaillant dans les équipes responsables de l'évaluation/orientation $(n=5)$ ou de l'application des mesures $(n=19)$ des deux centres jeunesse de Montréal (CJM-IU et les Centres de la jeunesse et de la famille Batshaw) ont participé à la recherche originale après avoir été approchés par leur chef de service. Ils ont choisi chacun deux dossiers d'enfants de minorités visibles, un dossier « exemplaire » (correspondant à une intervention réussie selon l'intervenant) et l'autre jugé « difficile » dont ils ont eu la charge dans les trois dernières années ( $n=48$ dossiers à l'étude). Cette méthode d'échantillonnage par cas extrêmes donne accès à un plus large éventail d'informations avec un nombre restreint de cas (Patton, 2002). Une certification éthique a été obtenue et une compensation financière a été remise aux fondations des deux centres jeunesse pour chaque entrevue réalisée dans le cadre de l'étude originale (analyses des entrevues non présentées ici).

\section{Instrument de collecte des données}

Cette étude s'intéresse à la description des signalements et de leur évaluation tels qu'ils sont consignés dans les dossiers des enfants. Une grille de 45 questions, ouvertes et fermées, permet d'organiser les

\footnotetext{
${ }^{1}$ La terminologie gouvernementale (Statistique Canada, 2017) avait été privilégiée afin de désigner des personnes membres de minorités ethnoculturelles plus susceptibles que les autres de faire l'objet d'une discrimination individuelle ou systémique sur la base de leur apparence physique ou de caractéristiques culturelles ( $p$. ex. : signes religieux) facilement reconnaissables (Kasper et Noh, 2001 ; Chan, 2008).
} 
informations colligées pour chaque dossier (voir Bousquet Saint-Laurent, 2013). Cette grille regroupe des informations générales pertinentes pour la recherche ( $p$. ex., durée d'implication de l'intervenant dans le dossier), ainsi que des informations sur l'identification de l'enfant visé par le signalement (langue parlée à la maison, origine ethnique, problèmes connus) et sur l'identification des membres de la famille, tels la fratrie (âge, lieu de résidence) et les parents (biologiques, conjoints ou tuteurs). Une section est également consacrée aux signalements antérieurs (décisions reliées aux signalements, dates des interventions); une autre, qui porte sur le signalement à l'étude, situe le contexte familial (lieu de résidence habituelle, personnes y vivant), la provenance du signalement (professionnel ou non) et le type de mauvais traitements motivant la rétention du signalement. Un résumé du signalement sous forme narrative est aussi fourni. Enfin, la dernière section de la grille concerne la vérification des faits (impact sur l'enfant, informations relatives aux attitudes des parents) et finalement, les décisions prises (faits avérés ou non et compromission établie ou non).

\section{Analyses}

Afin de répondre au premier objectif, le signalement et l'évaluation de tous les cas sont analysés transversalement (analyse intercas). Au moyen d'une méthode d'analyse thématique s'inspirant de Paillé et Mucchielli (2006), les contenus sont organisés en arborescence dans le logiciel NVivo 10. L'arbre thématique se construit à la lecture de l'ensemble des contenus issus de tous les dossiers et évolue en cours d'analyse. Deux exercices de contre-codage avec un second juge, sur un cinquième des contenus pris au hasard, permettent aussi de revoir certains codes en discutant et en visant un consensus. Au terme de l'analyse, chaque segment narratif apportant une idée est codé selon le thème auquel il se rattache. Une seconde analyse de ce contenu, maintenant réparti par thèmes, identifie tous les contenus relatifs à la diversité ethnoculturelle (p. ex. : croyances religieuses) ou à l'expérience migratoire ( $p$. ex. : déqualification professionnelle) (appelés ci-dessous « contenus ethnoculturels ou migratoires »).

Pour répondre au deuxième objectif concernant l'élaboration d'une typologie, une analyse verticale (intracas) inspirée de Dufour (2001) est réalisée. Chaque cas est d'abord résumé individuellement dans une vignette (portrait de la situation et de la famille). Par la suite, l'analyse des caractéristiques semblables et divergentes de l'ensemble des situations familiales conduit à des regroupements de profils familiaux distincts. Au terme de cette analyse, quatre profils familiaux émergent.

\section{RÉSUltats}

La première partie des résultats porte sur la description des contenus des signalements et sur leurs évaluations. Une deuxième partie décrit les quatre profils familiaux de la typologie. Dans les dossiers analysés, le signalement, c'est-à-dire les inquiétudes du signalant rapportées au DPJ quant à la sécurité et au bien-être d'un enfant, est consigné sous la forme d'un court rapport narratif d'un quart de page à une page. II est concis et rédigé au mode conditionnel par un analyste du centre de réception et de traitement des signalements. L'évaluation des signalements retenus ajoute des détails à ces premières inquiétudes. L'intervenant responsable de l'évaluation consulte les membres de la famille et différents acteurs impliqués auprès de celle-ci, puis consigne ces différentes informations au dossier sous forme de contenu narratif ou descriptif (questions objectives). Dans cette section également, les informations contenues dans les dossiers varient quant à leur quantité et à la diversité des sources, certaines étant très détaillées et d'autres, plus succinctes (une page à quelques pages de contenu narratif). Les contenus retrouvés dans les dossiers de ces enfants aux deux premières étapes du processus seront 
regroupés ici en cinq sections dont les quatre premières s'apparentent aux critères légaux encadrant la prise des décisions : les faits, les caractéristiques de l'enfant, les caractéristiques des parents et du milieu familial ainsi que les ressources dont dispose la famille (les informations dans les dossiers concernant chacun des critères pouvant se retrouver à divers endroits). Étant donné la particularité de l'échantillon, la dernière section décrit des contenus ethnoculturels ou migratoires identifiés dans l'ensemble des sections.

\section{Faits narrés dans les signalements et les évaluations}

La narration des faits rassemble des éléments de nature descriptive, qui portent notamment sur l'identité des personnes responsables, les formes de mauvais traitements, les circonstances (lieu des incidents, moment de la journée, événement particulier), la durée, la fréquence et le niveau de gravité, et sur lesquels se fonde la décision soit de mettre en place des mesures d'urgence, soit de poursuivre le traitement du dossier selon la procédure ordinaire. Les signalants qui interpellent la DPJ, des professionnels (associés aux milieux de la santé et des services sociaux, policier, de garde ou scolaire) dans l'exercice de leurs fonctions $(n=40)$ ou des non-professionnels $\left(n=7^{2}\right)$, rapportent des faits dont ils sont témoins ou des faits rapportés par un tiers (enfant lui-même, parent, personne qui côtoie la famille). Les faits décrits sont parfois observés ou observables et d'autres fois, plus hypothétiques, déduits d'indices observables, comme dans cet extrait: "Les policiers notent que le milieu est insalubre: pas de nourriture dans les armoires (sauf gruau et riz), pas de lits pour les enfants. Les policiers rapportent qu'il y a une bande élastique avec des nœuds accrochés à une porte qui pourrait servir à attacher les enfants. " L'évaluation permet de confronter la version des faits de plusieurs acteurs engagés dans la situation, d'experts ou d'autres intervenants. Parfois, en cours d'évaluation, les personnes interrogées mettent en lumière de nouveaux faits qui peuvent modifier l'interprétation de la situation (gravité, nature des mauvais traitements, personnes impliquées).

\section{Caractéristiques de l'enfant}

Les caractéristiques de l'enfant qui sont décrites sont en lien avec la situation de mauvais traitements et elles dressent un portrait général, par exemple en pointant des vulnérabilités comme l'âge, une dépendance aux soins, une condition de santé ou un retard de développement nécessitant une intervention particulière ou la mobilisation des parents. À l'évaluation, les intervenants recueillent plusieurs données descriptives concernant l'enfant et répertorient également des caractéristiques telles que des problèmes comportementaux (agressivité/impulsivité), émotionnels ou d'humeur, développementaux, de santé physique, d'attachement ou sociaux, un déficit cognitif, un diagnostic psychiatrique, l'abus de substances, etc.

Les conséquences des mauvais traitements sont rapportées lorsqu'elles sont observées par le signalant (blessures physiques, états ou changements comportementaux ou émotionnels) ou qu'elles lui sont confiées par un tiers, soit l'enfant lui-même (qui verbalise par exemple des appréhensions ou de la peur), un membre de la famille ou un autre intervenant (par exemple un enseignant ou un éducateur qui remarque des changements dans le comportement de l'enfant). La consultation d'experts de la santé permet de constater les conséquences physiques liées à des traumas ou à des irrégularités d'un traitement médical. L'avis d'autres professionnels et des intervenants impliqués auprès de l'enfant

\footnotetext{
${ }^{2}$ Nous n'avons pu obtenir l'information relative à la provenance du signalement pour un des dossiers à l'étude.
} 
dresse différents portraits de ses besoins spécifiques et des conséquences possibles des mauvais traitements sur son développement. Prenant en compte tous ces éléments, l'intervenant formule des hypothèses sur les conséquences à plus long terme des mauvais traitements pour l'enfant dans les différentes sphères développementales, comme dans cet exemple :

L'enfant est susceptible d'accuser un retard langagier et puisque le développement langagier dans la prime enfance est intimement relié au développement cognitif, aux capacités d'apprentissage et à l'acquisition des compétences sociales, ce contexte de discontinuité est porteur de risques importants pour le développement de l'enfant.

\section{Caractéristiques des parents et du milieu familial}

Les caractéristiques décrites ici concernent les personnes légalement responsables de l'enfant, qui tiennent le rôle de figure parentale ${ }^{3}$, mais qui peuvent aussi être des tuteurs avec ou sans lien biologique (p. ex. : fratrie, oncle, famille d'accueil). Les éléments rapportés sous ce thème sont surtout liés aux capacités parentales et aux difficultés personnelles des parents. Ces difficultés peuvent consister en des conflits conjugaux importants, des problèmes de santé physique ou de santé mentale, l'abus de substances et des activités criminelles. Des précisions sont également apportées sur les caractéristiques psychoaffectives des parents (p. ex. : fragilité émotive, sentiment de dépassement ou d'épuisement face à la situation). Des événements de vie marquants du parent ( $p$. ex. : grossesses antérieures, événements traumatiques dans l'enfance ou la vie adulte) et les compétences parentales telles qu'observées par l'intervenant sont aussi notés au dossier. Certains liens peuvent être faits entre leur histoire personnelle, des déclencheurs de crise et les difficultés présentes.

Plusieurs autres caractéristiques concernant le milieu familial sont rapportées. La structure familiale (monoparentale, biparentale intacte ou recomposée), la fratrie (nombre d'enfants, leur âge et leur lieu de résidence), la composition du foyer au lieu de résidence (autres adultes y vivant) et les sources familiales de stress comme l'instabilité résidentielle, la précarité financière ou des événements de vie marquants (p. ex.: maladie, décès, divorce, séparation, migration) en font partie. Les intervenants notent également au dossier des informations sur la répartition des rôles et responsabilités dans la famille, selon les verbalisations des parents ou les observations de l'intervenant.

\section{Ressources formelles et informelles du milieu}

Les informations sur les ressources sont dispersées dans l'ensemble des sections du signalement, selon le type de soutien offert et le membre de la famille qui en bénéficie. Le soutien formel désigne les interventions menées par les acteurs de différents organismes ou institutions impliqués auprès de la famille tels les milieux policier et hospitalier et les services de santé, les tribunaux et les services sociaux (communautaires comme des centres d'hébergement ou institutionnalisés comme les CLSC ${ }^{4}$ ), les interventions antérieures ou actuelles des services de protection de la jeunesse et celles d'autres services impliqués plus particulièrement auprès des enfants (milieu scolaire et milieu de garde). Les intervenants des différents services gravitant autour de la famille peuvent être sollicités pour corroborer des faits, pour contribuer à l'enrichissement du portrait familial en termes de forces et de besoins et

\footnotetext{
${ }^{3}$ Pour simplifier la lecture, le terme « parent » désigne les figures parentales qui sont chargées de l'enfant.

${ }^{4}$ Centre local de services communautaires (Québec).
} 
pour livrer des indications sur leur capacité de soutien et leur rôle auprès de la famille. Cet extrait décrit l'utilisation des ressources formelles par une mère, en qualifiant la durée et les résultats des interventions : "La mère a une longue histoire avec les ressources communautaires de Montréal. Elle a essayé d'obtenir de l'aide auprès de différentes ressources. Chaque fois, l'intervention a échoué et la famille est demeurée dans un état de chaos. "

Une autre dimension des ressources qui fait l'objet de descriptions est la qualité de la relation entre les parents, les membres de la famille et les intervenants de la DPJ ou des autres services. Les attitudes des parents peuvent être perçues comme défavorables à la collaboration (p. ex., conduites impulsives, agressives ou méfiantes). Certains parents expriment leur colère ou se rendent inaccessibles aux intervenants en refusant un suivi ou l'accès au domicile (absence aux rendez-vous, changement d'adresse sans en aviser l'intervenant). Les parents décrits comme " collaborants » reconnaissent au moins partiellement les difficultés et accueillent favorablement les interventions de la DPJ. Ils formulent des demandes d'aide ou acceptent l'aide qui leur est offerte et s'investissent dans les démarches proposées par l'intervenant.

Le soutien informel comprend entre autres l'aide offerte par des membres de la famille élargie, la communauté, des voisins ou des amis. De manière similaire, la présence ou l'absence de ce réseau ( $p$. ex., la famille élargie dans un autre pays ou un conjoint absent) sont notées. Les parents qualifient leur relation avec les membres de ce réseau (bonne relation, conflictuelle ou jugeante). Ils livrent aussi des indices sur les fonctions de ce réseau qui peuvent être instrumentales (soutien financier, ressources pour garder les enfants), émotionnelles (écoute, réconfort) ou informationnelles et normatives (conseils, information sur certains fonctionnements institutionnels). L'extrait suivant fait état de la qualité de la relation entre la mère et la grand-mère maternelle de l'enfant : " La mère considère que sa famille élargie est un milieu sécuritaire pour sa fille, mais ses relations avec sa mère sont très conflictuelles ; elle a maintenu avec elle un minimum de liens, car elle savait que cela était important pour [sa fille]. »

\section{Contenus ethnoculturels ou migratoires}

Ces contenus se retrouvent dans les sections narratives, plus souvent sous forme d'impressions, de propos rapportés ou d'histoires, ainsi que dans certaines questions objectives, comme le pays de naissance de l'enfant et de ses parents (s'ils sont nés à l'étranger, date d'arrivée et statut d'immigration), la religion pratiquée, l'appartenance ethnique et la langue parlée à la maison. Cette section permet de relever la nature du contenu ethnoculturel ou lié à l'expérience migratoire lorsqu'il est présent dans les dossiers. Dans les signalements, ces éléments sont presque inexistants (3 cas seulement sur 48 en font mention), tandis qu'à l'évaluation, ils sont présents dans 35 dossiers sur 48. Ces contenus sont présentés dans le tableau 1 selon les grands thèmes décrits plus haut.

Tableau 1 : Description des contenus ethnoculturels ou migratoires et exemples

\section{Contenus ethnoculturels ou migratoires selon les thèmes d'analyse}

Faits

- Circonstances ou explications des mauvais traitements en lien avec des croyances religieuses ou mystiques (tels la sorcellerie ou le vaudou); réinterprétation possible par les intervenants comme des indices de troubles de santé mentale.

\section{Extraits}

«Au niveau des soins de santé, Madame a de la difficulté à assumer le suivi et les coûts des soins, considérant son statut 


\begin{abstract}
- Faits nuancés par le contexte de précarité et d'adversité relié à une migration
précaire au niveau de

récente ou un statut d'immigration précaire, ainsi que par des différences

l'immigration. »

culturelles dans l'éducation ou les soins.
\end{abstract}

\title{
Caractéristiques de l'enfant
}

- Événements marquants du vécu, liés au parcours migratoire : événements prémigratoires ( $p$. ex., le décès de proches, la séparation avec des membres de la famille ayant émigré plus tôt, la violence ou des abus dans la famille et la préparation au départ), pendant la migration (p. ex., des séjours en camps pour réfugiés, le voyage) et post-migratoires (p. ex., l'intégration à la communauté, l'adaptation au milieu scolaire, ainsi que les répercussions des obstacles systémiques et des autres difficultés vécues par la famille).

- Verbalisation d'inquiétudes face à l'intervention, en lien avec les pratiques culturelles.

\section{Caractéristiques des parents et de la famille}

- Parcours migratoire et événements marquants (conditions familiales dans l'enfance, relation avec leurs propres parents, contexte disciplinaire et traumatismes).

- Difficultés post-migratoires : déqualification professionnelle, difficulté à trouver un emploi, perte de revenus, changement de statut social, obtention d'un statut d'immigrant ou de réfugié et autres stratégies d'adaptation engagées par les membres de la famille.

- La distribution des rôles et responsabilités dans la famille peut être liée à des valeurs ou à des modèles culturels traditionnels.

- L'accumulation des difficultés peut accroître des tensions conjugales déjà existantes.

\section{Ressources formelles}

- La perception des services, leur compréhension ou leur utilisation et la relation entre les intervenants et la famille peuvent être teintées par des schèmes ethnoculturels ou les expériences antérieures avec des figures d'autorité.

- Non-maîtrise de la langue comme obstacle à l'intervention.

« En entrevue, [l'enfant] pose des questions sur les familles d'accueil. Il dit que ce n'est pas pour lui, car il est musulman. Il doit vivre dans un environnement différent des autres jeunes et avoir une alimentation particulière. »

« À son arrivée, [la mère] a donc été choquée de voir que le père n'avait pas d'emploi et vivait dans un appartement insalubre. La famille vivait d'importantes difficultés financières. Madame regrette d'être venue au Québec et elle ne comprend toujours pas pourquoi le père l'a fait venir ici. »

« Madame n'arrive pas à comprendre le sens de notre intervention et elle croit que nous nous "acharnons" sur elle et que nous sommes racistes. "

\section{Ressources informelles}

- Réseau de soutien resté au pays, dans certains cas absent et dans d'autres, implication à distance (p. ex., trouver un conjoint après un divorce, prendre en charge des enfants plus difficiles ou ceux laissés au pays, entretenir des attentes par rapport à la réussite sociale et financière).

- Les membres du réseau dans le pays d'accueil, dont la communauté ethnique ou religieuse ou la famille élargie, s'impliquent en donnant des conseils sur les relations conjugales ou l'éducation des enfants, ou exercent une influence ou une forme de pression sociale.

« Elle est isolée familialement et socialement, n'ayant aucune famille ou ami au pays afin de la supporter [...]. Madame a quatre autres enfants qui vivent [dans son pays d'origine] et qui sont confiés à sa famille élargie. »

\section{Typologie des familles signalées}

L'analyse permet finalement de distinguer quatre profils parmi les familles issues de la diversité culturelle faisant l'objet de signalements aux services de protection. Le premier profil est celui des familles traversant une crise, dont les difficultés semblent plus transitoires que celles des autres profils. 
Typiquement, il s'agit d'un premier signalement, provenant exclusivement de professionnels. Les difficultés de l'enfant sont aussi identifiées par les parents, qui ont une bonne relation d'attachement avec leur enfant. Les parents ont aussi les capacités de se mobiliser durant la crise pour apporter des changements et solliciter l'aide d'intervenants (en participant activement aux moyens d'action proposés), et ce, malgré leurs difficultés personnelles. Le réseau informel peut aussi soutenir les parents dans leur rôle lorsqu'il est présent. Enfin, certains éléments de crise sont expliqués par une migration récente ( $p$. ex., précarité financière, difficultés à s'insérer sur le marché de l'emploi, déqualification professionnelle, choc culturel).

Le second profil présente des familles qui abdiquent leur rôle parental, dont les parents, exclusivement des mères monoparentales, n'arrivent pas ou plus, en raison de difficultés personnelles, à répondre adéquatement aux besoins de leur enfant. Face à la situation de crise ou à l'intervention de la DPJ, elles reconnaissent leurs difficultés et celles que peut vivre leur enfant. Elles ne se sentent pas capables d'apporter les changements nécessaires au bien-être des enfants et acceptent ainsi les interventions de la DPJ, qui visent notamment le placement dans un autre milieu de vie. Le réseau informel peut être présent et disponible pour prendre en charge l'enfant. Cet extrait illustre ce type de résolution de la situation: "La mère a effectivement collaboré et considérait que le milieu de vie de la grand-mère maternelle était un lieu de placement approprié pour [son fils]. » Lorsque des contenus ethnoculturels ou migratoires sont abordés, ils peuvent jeter un éclairage sur des événements de l'histoire personnelle de ces mères qui ont contribué à leurs difficultés actuelles.

Le troisième profil regroupe les familles isolées, qui vivent leur histoire familiale loin des ressources formelles et de certaines ressources informelles. Les difficultés que vivent les membres de la famille ne sont pas perçues ou reconnues par les parents. Les enfants sont considérés comme très vulnérables, en termes de besoins et de conséquences observées par les intervenants, et les parents présentent de nombreuses difficultés personnelles. Cependant, les parents ne sollicitent pas l'aide des ressources formelles, la rejettent même parfois, et font de même avec certaines personnes de leur réseau informel. Lorsque le réseau informel est impliqué, les intervenants le perçoivent comme entravant leur intervention (contribuant parfois à un isolement communautaire). Lorsque des éléments ethnoculturels ou migratoires sont abordés, ils concernent des différences sur le plan de la compréhension des besoins des enfants ou de la famille, parfois une barrière linguistique et l'influence d'une communauté sur les attitudes et les priorités parentales.

Le dernier profil est celui des familles éclatées, dont les milieux familiaux sont décrits comme chaotiques, sans organisation ni stabilité. Typiquement, pour ces familles, la crise dure depuis plusieurs années, il ne s'agit pas d'un premier signalement et les interventions mises en place n'ont pas permis de résoudre la situation. Les répercussions des mauvais traitements sur le développement des enfants sont jugées importantes. Les membres de la famille immédiate ont aussi des problèmes personnels majeurs, qui amènent l'implication de plusieurs ressources institutionnelles et communautaires, qui les rendent parfois absents du milieu familial ( $p$. ex., milieux policiers et carcéraux, centres d'hébergement et de désintoxication, urgences médicales, centres sociaux et DPJ). Les parents sont décrits par les intervenants de la DPJ comme non coopératifs et comme n'ayant pas les capacités ou la motivation nécessaires pour fournir une réponse adaptée aux besoins des enfants. Ces familles ne semblent pas avoir de réseau social établi. Même si elles recourent parfois à des relations pour des dépannages ponctuels, elles ne développent pas de relations significatives avec leur entourage. Enfin, les éléments ethnoculturels ou migratoires abordés dans ce profil renvoient à des aspects de l'histoire migratoire de 
la famille (séparations et réunions familiales), ainsi qu'à une précarité des statuts d'immigration.

\section{Discussion}

\section{Faits saillants}

Les contenus des signalements et de leur évaluation se rapportent principalement aux critères reliés à la compromission fixés par la LPJ. Toutefois, il existe de grandes disparités entre les dossiers, touchant autant la quantité d'informations (concernant la famille, l'enfant ou le réseau) que la qualité (points de vue de différents experts et intervenants rapportés ou éléments d'analyse). Les familles qui ont fait l'objet d'interventions antérieures de la part des services de protection ou d'autres services psychosociaux et de santé présentent des dossiers plus étayés. Dans une recherche de Lavergne et al. (2014) sur la disproportion des enfants issus de groupes racisés dans le système de la protection de la jeunesse, les intervenants évoquent des contraintes comme le temps limité pour faire l'évaluation des situations en selon les exigences légales et une charge de travail importante considérant le nombre croissant d'enfants nécessitant des interventions de la DPJ (voir CJM-IU, 2014), ce qui pourrait expliquer une partie des disparités.

Une différence liée au cadre légal est le classement des énoncés concernant la collaboration entre les parents et les intervenants de la DPJ. Comme mentionné en introduction, un des critères sur lesquels se fonde la prise de décision concerne la capacité et la volonté des parents de mettre fin à la situation de compromission. La capacité et la volonté telles qu'évaluées par les intervenants concernent les attitudes et les comportements des personnes responsables vis-à-vis des faits allégués et des services et se traduisent dans la reconnaissance des problèmes, la motivation à résoudre les problèmes et la collaboration avec les services. Ces aspects s'apparentant davantage à ceux qui se rapportent à la réaction des parents face à l'intervention des services de protection, ils sont analysés avec les ressources du milieu.

Les contenus ethnoculturels ou migratoires varient aussi énormément d'un dossier à l'autre, puisque certains peuvent contenir plusieurs éléments et d'autres, absolument aucun. Une des pistes explicatives est la nature de l'échantillon, qui regroupe des enfants identifiés comme appartenant à une minorité visible par les intervenants. Bien que les statistiques démontrent des difficultés communes aux personnes appartenant à ce groupe, comme une plus grande précarité d'emploi à scolarité égale et un revenu moyen plus faible, qui maintiennent plusieurs ménages dans la pauvreté et entraînent d'autres problèmes connexes (Statistique Canada, 2006), ces personnes ont aussi des parcours et des vulnérabilités différentes si elles sont primo arrivantes, issues de l'immigration ou citoyennes canadiennes depuis plus de deux générations (Hassan et al., 2011 ; Dettlaff et Earner, 2012).

De plus, les contenus ethnoculturels ou migratoires sont intégrés à une analyse de la situation dans quelques cas seulement. Certains auteurs mettent en avant l'importance d'une compétence et d'une sensibilité culturelles dans l'intervention. Une connaissance plus globale et plus complète de la situation ainsi que l'établissement d'une relation de confiance dans les premiers contacts entre les intervenants et la famille favorisent un meilleur arrimage entre les besoins et les interventions (Fontes, 2005 ; Maiter et Stalker, 2011 ; Dufour et al., 2012). Certains auteurs travaillant sur le sujet suggèrent l'utilisation d'outils adaptés aux familles issues de la diversité ethnoculturelle, comme des grilles pour soutenir l'évaluation culturellement sensible (Fontes, 2005 ; Pine et Drachman, 2005), le développement de partenariats avec des organismes de la communauté ou des rencontres du cercle familial (Family Group 
Conference), un outil de communication et d'intervention favorisant la participation et l'implication de plusieurs membres de la famille et plusieurs intervenants gravitant autour de celle-ci (Dettlaff et Rycraft, 2008). Toutefois, peu d'évaluations ont été menées sur l'efficacité des outils et des actions permettant de soutenir l'intervention, ce qui ne favorise pas un consensus autour des meilleures pratiques en protection de la jeunesse auprès de ces familles (Dettlaff et Rycraft, 2008 ; Dufour et al., 2017). D'autres auteurs mettent aussi en garde contre une " culturalisation » des faits, qui oriente les décisions sans toujours correspondre aux besoins de la famille et de l'enfant, tendant à offrir des solutions qui ne favorisent pas le changement (Hassan et Rousseau, 2007 ; Dufour et al., 2012). II est important de tendre vers un équilibre pouvant faire écho à la sensibilité culturelle tout en gardant en tête la protection des enfants.

D'autres recherches auprès des intervenants de la protection soulèvent des difficultés organisationnelles et systémiques liées à l'évaluation auprès de certaines familles provenant de la diversité ethnoculturelle (plus particulièrement celles issues d'une immigration récente), comme le recours à des interprètes (obtention du service, planification des rencontres selon la disponibilité d'un autre acteur et temps supplémentaire à allouer pour les entretiens ou choix de certaines pertes d'information), l'accessibilité à de la formation, à des personnes ressources ou à des consultations cliniques pour des cas plus difficiles, la méconnaissance des services et la différence par rapport aux attentes développementales qui nécessitent plus de temps pour expliquer le rôle de l'intervenant et les aspects légaux ou plus d'échanges sur les conceptions de chacun des besoins des enfants et des rôles parentaux (Hassan et Rousseau, 2007 ; Lavergne et al., 2014).

\section{Typologie des familles}

Les profils familiaux proposent une image de ces familles aux premiers contacts avec les services de protection. Ce qui distingue principalement les quatre profils familiaux est, d'une part, la gravité des mauvais traitements (sévérité, chronicité, fréquence et conséquences pour l'enfant) et, d'autre part, la collaboration au sens des critères d'analyse légaux (capacité et volonté des parents). Ces deux dimensions influencent grandement la prise de décision quant à la compromission et aux mesures d'intervention.

II existe peu de typologies des familles impliquées dans l'intervention de la DPJ (pour des analyses centrées sur les personnes, voir les travaux de Gagné et Bouchard, 2004, et Dufour et al., 2011), les recherches sur la violence envers les enfants s'intéressant plutôt aux caractéristiques des enfants et des parents par type de mauvais traitements (Higgins, 2004 ; Mayer et al., 2007) et aux analyses centrées sur les variables. Certaines recherches axées sur les personnes se rapprochent toutefois davantage des modèles d'évaluation et d'analyse écosystémiques souvent recommandés pour les interventions en protection de la jeunesse, et particulièrement auprès des familles migrantes ou issues de la diversité ethnoculturelle (Fontes, 2005). Higgins (2004) propose une classification selon la gravité des mauvais traitements (fréquence/sévérité), remettant en question le classement plus répandu par type de mauvais traitements, soutenant entre autres arguments que ces différentes formes de violence sont souvent entremêlées dans les dynamiques des familles vivant des difficultés importantes, augmentant du même coup la gravité des conséquences sur les enfants. La gravité des situations, considérée dans la présente typologie, semble en effet une piste intéressante pour décrire les familles et évaluer les besoins en intervention. Les familles traversant une crise semblent plus « faciles » à aider que les familles éclatées, dont la structure inexistante, les difficultés et leurs impacts importants pour 
tous les membres induisent une « fragilité » dont il apparaît plus difficile de se relever. Cependant, cette seule dimension de la gravité des mauvais traitements ne suffit pas à éclairer l'ensemble de la situation et ne permet pas de déceler les facteurs de protection et les leviers d'intervention présents dans l'environnement familial et élargi. La collaboration ou l'alliance thérapeutique entre les parents et les intervenants constitue ainsi selon plusieurs auteurs un facteur clé de l'intervention et son absence est perçue comme un obstacle important à l'amélioration du bien-être de l'enfant qui se retrouve pris dans des conflits entre les intervenants et la famille (Hassan et Rousseau, 2007). À partir des données du même projet de recherche original que celui dont est tirée la présente analyse secondaire, Couture (2012) a élaboré une typologie de la collaboration des familles de minorités visibles avec les intervenants de la protection en lien avec leur réseau informel. Elle en dégage six dynamiques familiales couplées avec les compétences professionnelles déployées. La collaboration des parents est assurément un enjeu important pour la réussite de l'intervention visant à assurer le bien-être de l'enfant. Cette approche des dynamiques familiales permet aussi de mettre en lumière d'importants acteurs gravitant autour de la famille (nuisibles ou aidants pour l'intervenant de protection) qui peuvent faire partie des solutions, comme dans le cas des familles qui abdiquent leur rôle parental.

\section{Limites et implications}

Quelques limites concernent les données et l'échantillon. Cette recherche étant une analyse secondaire, les données recueillies ne visent pas spécifiquement à répondre à ses objectifs. Bien que la méthode d'échantillonnage par cas extrêmes permette de couvrir un large éventail de situations, après l'analyse des 48 situations d'enfants, la saturation empirique ne semble pas être atteinte pour la typologie (Pires, 1997), c'est-à-dire qu'il pourrait y avoir d'autres situations familiales qui ne soient pas représentées dans ces profils. Les données figurant dans les dossiers sont consignées par les intervenants de la protection et d'autres experts consultés, ce qui teinte les profils familiaux des considérations légales et de l'opinion professionnelle des différents intervenants. De plus, même en ayant un échantillon constitué de familles appartenant toutes à une minorité visible selon les intervenants, les réalités et les parcours de ces familles peuvent être très différents. Enfin, cette recherche ne permet pas la comparaison du contenu des dossiers de ces familles avec celui des dossiers des autres familles signalées à la protection de la jeunesse.

Les résultats de cette étude bénéficieraient d'une recherche complémentaire, à partir d'un échantillon plus grand, comprenant plus d'aspects des dynamiques familiales et dont les données seraient systématiquement recueillies pour l'ensemble des familles afin d'étoffer davantage les portraits dégagés. Une validation de ces profils familiaux auprès des intervenants de la protection de la jeunesse permettrait également d'évaluer la correspondance avec de nouveaux cas et la pertinence de la typologie pour la pratique. Des renseignements supplémentaires sur les besoins et les dynamiques propres aux familles issues de la diversité ethnoculturelle pourraient être systématiquement recueillis à l'aide de grilles. L'inclusion de ces informations pour l'analyse de la situation familiale, et ce, dès les premières étapes d'évaluation du signalement, influencerait peut-être la trajectoire des familles dans les services sociaux. Ces éléments ethnoculturels et du parcours migratoire pourraient également permettre d'identifier des leviers et de cibler des interventions correspondant davantage à certains besoins des familles. 


\section{Conclusion}

La littérature nous renseigne sur l'importance du contexte familial dans les interventions de protection et tout particulièrement pour les familles issues de la diversité ethnoculturelle (Fontes, 2005 ; Dettlaff et Rycraft, 2008 ; Maiter et Stalker, 2011). Cependant, plusieurs barrières peuvent entraver l'action des intervenants et le bien-être des enfants et de leur famille. Les portraits familiaux qui ressortent de cette analyse montrent la complexité des conséquences liées à la gravité des situations de mauvais traitements à l'égard des enfants. Cette gravité émerge à la fois de la nature, de l'intensité et de la fréquence des mauvais traitements envers l'enfant, des problèmes personnels des membres de la famille (conséquences des mauvais traitements sur l'enfant, difficultés connues des parents et des autres membres de la famille, relations avec les ressources formelles et informelles), ainsi que de la capacité et de la volonté des parents à s'investir dans la démarche proposée par la DPJ. À la lumière de cette analyse, ainsi que d'autres recherches sur le sujet, on constate qu'il importe de se pencher sur les pratiques d'organisations telles que la protection de la jeunesse pour répondre aux besoins des familles les plus vulnérables de la société en adéquation avec leur réalité, leurs forces et leurs défis. II faut également continuer de se questionner sur l'impact plus large de nos pratiques dans les services aux familles, en considérant également nos politiques familiales (p. ex., concept de famille élargie, logements adaptés aux différents types de familles) et d'immigration ( $p$. ex., statut d'immigration, reconnaissance de la formation et de l'expérience de travail à l'étranger), puis des valeurs et des normes sociétales (p. ex., les préjugés et la discrimination à l'égard des minorités culturelles, l'acceptation d'un métissage culturel). Idéalement, les efforts mis dans l'intervention devraient parallèlement se retrouver dans les pratiques préventives de la maltraitance pour les personnes vulnérables et dans le développement de pratiques permettant une alliance entre le milieu familial et les intervenants des différentes ressources aux familles, afin de favoriser le bien-être et le développement de l'enfant.

BOUSQUET SAINT-LAURENT, Tanya, M. Sc. Psychoéducation

École de psychoéducation

Université de Montréal

DUFOUR, Sarah, Ph. D. Psychologie, Professeure agrégée

École de psychoéducation

Université de Montréal

\section{RÉFÉRENCES}

Besharov, D. J. (2005). Overreporting and underreporting of child abuse and neglect are twin problems. Dans D. R. Loseke, R. J. Gelles et M. M. Cavanaugh (dir.), Current controversies on family violence (p. 285-298). Thousand Oaks, CA : Sage Publications.

Bibby, R. W. et Grenville, A. (2016). What the Polls Do Show: Toward Enhanced Survey Readings of Religion in Canada. Canadian Review of Sociology, 53(1), 123-136.

Bibby, R. W., et Reid, A. (2015). The 2015 religion survey. Vancouver, Canada : Angus Reid Institute. 1975, 1985, 2000 - Reginald W. Bibby, Project

Bousquet Saint-Laurent, T. (2013). Analyse qualitative du contenu des signalements retenus à la 
protection de la jeunesse et leur évaluation pour les enfants de minorités visibles. Mémoire de maîtrise (Psychoéducation), Université de Montréal.

Centre jeunesse de Montréal - Institut universitaire (CJM-IU) (2014). S'investir pour le futur. Rapport annuel de gestion 2013-2014 (78 p.).

<http://www.centrejeunessedemontreal.qc.ca/pdf/cjm/rapport_annuel_2014.pdf>.

Chan, A. (2008). Every child matters? A critical review of child welfare reforms in the context of minority ethnic children and families. Child Abuse Review, 17(1), 6-22.

Conseil des relations interculturelles. (2007). Avis sur la prise en compte et la gestion de la diversité ethnoculturelle. Québec : Conseil des relations interculturelles.

Couture, D. (2012). La collaboration des parents de minorités visibles avec les services de la protection de la jeunesse : le rôle du soutien informel parental et des compétences de l'intervenant. Mémoire de maîtrise (Psychoéducation), Université de Montréal.

Derezotes, D., et J. Poertner (2005). Factors contributing to the overrepresentation of African American children in the child welfare system. Dans D. Derezotes, M. Testa et J. Poertner (dir.), Race matters in child welfare: The overrepresentation of African American children in the system (p. 1-23). Washington, DC : CWLA Press.

Dettlaff, A. J., et I. Earner (2012). Children of immigrants in the child welfare system: Characteristics, risk, and maltreatment. Families in Society: The Journal of Contemporary Social Services, 93(4), 295-303. <http://doi.org/10.1606/1044-3894.4240>

Dettlaff, A. J., et J. R. Rycraft (2008). Deconstructing disproportionality: Views from multiple community stakeholders. Child Welfare, 87(2), 37-59.

Dufour, S. (2001). La santé mentale des enfants de milieux défavorisés conceptions, pratiques et profils de pères. Thèse de doctorat (Psychologie), Université du Québec à Montréal.

Dufour, S., G. Hassan et C. Lavergne (2012). Mauvais traitements et diversité culturelle : bilan des connaissances et implications pour la pratique. Dans M.-H. Gagné, S. Drapeau et M.-C. SaintJacques (dir.), Les enfants maltraités : de l'affliction à l'espoir. Pistes de compréhension et d'action (p. 143-171). Québec : Presses de l'Université Laval.

Dufour, S., C. Lavergne et Y. Ramos (2015). Relations spatiales entre les caractéristiques des territoires et les taux d'enfants de groupes ethnoculturels signalés à la protection de la jeunesse. Revue canadienne de santé publique, 106(7), Supplément 2, eS21-eS30.

Dufour, S., M.-Ė. Clément, C. Chamberland et D. Dubeau (2011). Child abuse in a disciplinary context: A typology of violent family environments. Journal of Family Violence, 25(4), 357-448. <http://doi.org/10.1007/s10896-011-9394-0>

Dufour, S., C. Lavergne, T. Bousquet St-Laurent et M. Lord (2017). Bilan et efficacité des pratiques 
auprès des enfants issus de la diversité ethnoculturelle en protection de la jeunesse. Défi jeunesse, 23(2), 53-61.

Dufour, S., C. Lavergne, J. Gaudet et D. Couture (2016). Protecting visible minority children: Familycaseworker dynamics and protective authority intervention strategies. Canadian Psychology, 57(4), p. 356-364.

Eid, P., J. Magloire et M. Turenne (2011). Profilage racial et discrimination systémique des jeunes racisés. Rapport de la consultation sur le profilage racial et ses conséquences (126 p.). Québec: Commission des droits de la personne et de la jeunesse.

<http://www.cdpdj.qc.ca/publications/profilage_rapport_fr.pdf>

Finkelhor, D. (2005). The main problem is underroporting child abuse and neglect. Dans D. R. Loseke, R. J. Gelles et M. M. Cavanaugh (dir.), Current controversies on family violence (p. 299-309). Thousand Oaks, CA : Sage Publications.

Fluke, J., B. Jones Harden, M. Jenkins et A. Ruehrdanz (2011). Disparities and disproportionality in child welfare: Analysis of the research. <http://www.cssp.org/publications/childwelfare/alliance/Disparities-and-Disproportionality-in-Child-Welfare_An-Analysis-of-the-ResearchDecember-2011.pdf>

Fontes, L. A. (2005). Child abuse and culture: Working with diverse families. New York, NY : The Guilford Press.

Gagné, M.-H., et C. Bouchard (2004). Family dyamics associated with the use of psychologically violent parental practices. Journal of Family Violence, 19(2), 117-130.

<http://doi.org/10.1023/B:JOFV.0000019842.74408.b0>

Gouvernement du Québec (2009). Loi sur la protection de la jeunesse. Québec. <http://www.cdpdj.qc.ca/fr/commun/docs/LPJ.pdf>

Hassan, G. et Rousseau, C. (2007). La protection des enfants : enjeux de l'intervention en contexte interculturel. Association pour la recherche interculturelle, 45, 37-50.

Hassan, G., B. D. Thombs, C. Rousseau, L. J. Kirmayer, J. Feightner, E. Ueffing et K. Pottie (2011). Appendix 12: Child maltreatment: evidence review for newly arriving immigrants and refugees (15 p.). Doi:10.1503/cmaj.090313

Higgins, D. J. (2004). The importance of degree versus type of maltreatment: A cluster analysis of child abuse types. The Journal of Psychology, 138(4), 303-324.

Hill, R. B. (2006). Synthesis of research on disproportionality in child welfare: An update. Washington, DC : Casey-CSSP Alliance for Racial Equity in the Child Welfare System.

Hirani, K., D. Payne, R. Mutch et S. Cherian (2016). Health of adolescent refugees resettling in highincome countries. Archives of Disease in Childhood, 101(7), 670-676. 
Institut national d'excellence en santé et services sociaux (2016). Les mauvais traitements psychologiques : un mal silencieux. Bilan des directeurs de la protection de la jeunessedirecteurs provinciaux 2016. Québec : Gouvernement du Québec.

<https://www.inesss.qc.ca/fileadmin/doc/INESSS/ServicesSociaux/Bilan DPJ/INESSS Bilan DPJ Mauvais traitements psycho 2016.pdf>

Kaspar, V., et S. Noh (2001). Discrimination and identity: An overview of theoretical and empirical research. Department of Canadian Heritage for the Ethnocultural, Racial, Religious, and Linguistic Diversity and Identity Seminar, Halifax, Nouvelle-Écosse. $<$ http://canada.metropolis.net/events/ethnocultural/index e.html>

Lavergne, C., S. Dufour et D. Couture (2014). Point de vue des intervenants sur la disproportion des enfants issus de groupes racialisés dans le système de protection de la jeunesse : causes et pistes de solution. Alterstice - Revue internationale sur la recherche interculturelle, 4(1), 17-30.

Lavergne, C., S. Dufour, J. Sarmiento et M.-Ė. Descôteaux (2009). La réponse du système de protection de la jeunesse montréalais aux enfants issus des minorités visibles. Intervention, 131, 233-241.

Lavergne, C., S. Dufour, N. Trocmé et M.-C. Larrivée (2008). Visible minority, Aboriginal, and Caucasian children investigated by Canadian protective services. Child Welfare, 87(2), 59-76.

LeBrun, A., G. Hassan, M. Boivin, S.-L. Fraser, S. Dufour et C. Lavergne (2015). Review of child maltreatment in immigrant and refugee families. Revue canadienne de santé publique, 106(7), Supplément 2, eS45-eS57.

Maiter, S., et C. Stalker (2011). South Asian immigrants' experience of child protection services: Are we recognizing strengths and resilience? Child \& Family Social Work, 16(2), 138-148. <http://doi.org/10.1111/j.1365-2206.2010.00721.x>

Mayer, M., C. Lavergne, M. Tourigny et J. Wright (2007). Characteristics differentiating neglected children from other reported children. Journal of Family Violence, 22(8), 721-732. <http://doi.org/10.1007/s10896-007-9120-0>

Millett, L. S. (2016). The healthy immigrant paradox and child maltreatment: A systematic review. Journal of Immigrant and Minority Health, 18(5), 1199-1215.

Ministère de l'Immigration, de la Diversité et de l'Inclusion (2016). Immigrants selon la catégorie d'immigrants, Québec, 1980-2015. <http://www.stat.gouv.qc.ca/statistiques/populationdemographie/migration/internationales-interprovinciales/605.htm $>$

Ministère de la Santé et des Services sociaux (2017). Bilan des DPJ et DP 2017. L'adolescence, une traversée en eaux vives. Données montréalaises. <https://www.ciussscentresudmtl.gouv.qc.ca/actualites/actualite/bilan-des-dpj-dp2017/0b6b16b68b459422e836c6e4cd6757c0/> 
Paillé, P., et A. Mucchielli (2006). L'analyse qualitative en sciences humaines et sociales ( $2^{\mathrm{e}}$ éd.). Paris : Armand Colin.

Patton, M. Q. (2002). Qualitative research \& evaluation methods (3 éd.). Thousand Oaks, CA : Sage Publication.

Pine, B. A., et D. Drachman (2005). Effective child welfare practice with immigrant and refugee children and their families. Child Welfare, 84(5), 537-562.

<http://www.ncbi.nlm.nih.gov/pubmed/16435650>

Pires, A. P. (1997). Échantillonnage et recherche qualitative : essai théorique et méthodologique. Dans J. Poupart (dir.), La recherche qualitative : enjeux épistémologiques et méthodologiques (p. 113169). Montréal : Gaëtan Morin, p. 113-169.

Pottie, K., G. Dahal, K. Georgiades, K. Premji et G. Hassan (2015). Do first generation immigrant adolescents face higher rates of bullying, violence and suicidal behaviours than do third generation and native born? Journal of Immigrant and Minority Health, 17(5), 1557-1566.

Sarmiento, J., et C. Lavergne (2017). Les enfants et les jeunes des minorités ethnoculturelles en protection de la jeunesse et dans les services aux jeunes contrevenants : portrait des usagers et de leur trajectoire de services. Défi jeunesse, 23(2), 8.

Statistique Canada (2006). La mosaïque ethnoculturelle du Canada, Recensement de 2006 : principales régions métropolitaines de recensement du Canada. <http://www12.statcan.ca/censusrecensement/2006/as-sa/97-562/p19-fra.cfm>

Statistique Canada (2013). Immigration et diversité ethnoculturelle au Canada. Enquête nationale auprès des ménages, 2011. <http://publications.gc.ca/pub?id=9.641715\&sl=1>

Tardif-Grenier, K., C. Lavergne et J. Sarmiento (2016). Diversité culturelle et violence agie ou subie par les jeunes : bilan de la recherche québécoise des quinze dernières années. Alterstice - Revue internationale sur la recherche interculturelle, 6(2), 41-58.

Tétart, F. (2015). Atlas des religions. Passions identitaires et tensions géopolitiques (p. 63). Paris : Éditions Autrement, coll. «Atlas Monde ».

Ville de Montréal (2013). Population des minorités visibles : des différences marquées entre l'île de Montréal et la couronne urbaine.

<http://ville.montreal.qc.ca/pls/portal/docs/PAGE/MTL_STATS_FR/MEDIA/DOCUMENTS/POPULA TION_ET_D\%C9MOGRAPHIE_MINORIT\%C9S.PDF> 